\title{
Corela
}

Cognition, représentation, langage

HS-33 | 2020

Textuel, textiel. Repenser la textualité numérique

\section{La « rédaction web » : normes, contextes, textualités}

Note de recherche

\section{Émilie Née}

\section{(2) OpenEdition}

\section{Journals}

\section{Édition électronique}

URL : https://journals.openedition.org/corela/11888

DOI : $10.4000 /$ corela. 71888

ISSN : $7638-573 X$

\section{Éditeur}

Cercle linguistique du Centre et de l'Ouest - CerLICO

\section{RÉFÉRENCE ÉLECTRONIQUE}

Émilie Née, «La "rédaction web » : normes, contextes, textualités », Corela [En ligne], HS-33 |

2020, mis en ligne le 24 septembre 2020, consulté le 13 juillet 2021. URL : http://

journals.openedition.org/corela/11888; DOI : https://doi.org/10.4000/corela.11888

Ce document a été généré automatiquement le 13 juillet 2021.

\section{c) (7)(2)}

Corela - cognition, représentation, langage est mis à disposition selon les termes de la licence Creative Commons Attribution - Pas d'Utilisation Commerciale - Partage dans les Mêmes Conditions 4.0 International. 


\section{La « rédaction web » : normes, contextes, textualités}

Note de recherche ${ }^{1}$

Émilie Née

\section{Contexte questionnement}

1 Avant de commencer, nous souhaitons prévenir le lecteur que cette note de recherche prend pour point de départ un certain nombre d'observations empiriques. Son ancrage est celui de l'analyse du discours, envisagée comme discipline interprétative et lecture critique des énoncés mais aussi comme démarche susceptible d'apporter sa contribution à une didactique de l'écrit. Notre réflexion se situe ainsi dans le sillage de travaux en analyse du discours sur l'écriture en contexte professionnel (Rinck et Sitri 2012, Laborde-Milaa et al. 2014, Gournay et Née 2016) et sur des genres d'écrits routiniers (Adam 1997, 2011, Maingueneau 1998). Compte tenu de son objet, les textes produits dans le cadre de la rédaction web, elle s'inspire de plusieurs approches de la textualité numérique, du côté des sciences du langage (Paveau 2015, 2017, De Angelis 2018, Baudouin 2014) et des sciences de l'information et de la communication (travaux du Gripic, notamment).

2 Depuis 2012, nous sommes amenée à former, au niveau de la licence ou du master, des étudiants dont un certain nombre réalisent des stages ou s'orientent vers les métiers de la rédaction web (appelée aussi webrédaction). Au cours des suivis de stage que nous avons assurés, nous avons eu un accès relativement privilégié (mais encore très partiel) à la fabrique de "textes ${ }^{2}$ » destinés au web et ce dans différentes sphères d'activité (tourisme, culture, alimentation, vin, grande distribution, infodivertissement, industrie du luxe, radio/journalisme d'information, formation). Nous avons alors été sensible au poids important de normes rédactionnelles communes liées à la textualité numérique, en même temps qu'à une diversité de conditions de production de ces écrits et, avec elles, de pratiques rédactionnelles, ces différents aspects affectant les écrits produits. Cette diversité semble liée aux vocations de ces textes, au modèle économique de 
l'entreprise ou de l'institution qui les produit ou encore à la représentation qu'en ont les acteurs de l'entreprise.

Dans un objectif de formation et de connaissance, mais aussi dans un objectif cumulatif et critique par rapport à la littérature professionnelle existante sur la rédaction web, nous souhaitons aujourd'hui livrer quelques premiers jalons et éléments de réflexion pour la caractérisation discursive de ces textes. Nous prenons en compte pour cela à la fois la composante discursive de ces productions et les discours construisant la rédaction web comme pratique d'écriture spécifique, ces discours asseyant un ensemble de normes, diffusant des pratiques, avec lesquelles doivent composer les scripteurs.

De plus, nous nous intéressons non seulement aux écrits produits mais aussi au processus de production, qui nous semble un observatoire précieux pour mettre au jour des composantes (techno)linguistiques et (techno)langagières de ces textes, ainsi qu'un certain nombre de contraintes et de déterminations expliquant la présence de certaines formes linguistiques et discursives.

Plusieurs questions guideront cette réflexion. Quels (nouveaux) observables sont intéressants à prendre en compte, dans une perspective de formation et dans une perspective de compréhension / de saisie discursive de ces écrits d'un nouvel ordre ? Quel système de "règles invisibles » (Maingueneau 2012, cité par Paveau 2017) peut-on mettre au jour dans les écrits produits par les personnes occupant la fonction de rédacteur web ? Observe-t-on des "conflits de normes" (Devriendt 2017)? Si oui, à quels niveaux se jouent-ils?

\section{L'écrit et le texte du point de vue de la rédaction web}

6 Les discours professionnels définissant la rédaction web et le métier de rédacteur web sont éclairants en ce qu'ils donnent accès à des représentations du texte, à une culture partagée sur le numérique (De Angelis 2018) ainsi qu'à des normes que l'on retrouve dans les entreprises ${ }^{3}$. Il s'agit de discours que l'on recueille sur des sites spécialisés dans le web, la formation, et enfin dans des manuels ou guides pratiques. Souvent nourris d'une pratique de la textualité numérique ${ }^{4}$, ces discours dessinent les fonctions du scripteur et vont peser sur les textes produits et les formes langagières privilégiées.

\subsection{Une pratique d'écriture polyvalente et distribuée dans de nombreux domaines}

Le rédacteur web est un professionnel chargé de fournir un contenu textuel pour les sites Internet. [...]

Le rédacteur web peut travailler aussi bien dans une entreprise de communication, une entreprise de référencement que dans toutes autres entreprises disposant d'un site web. Il peut aussi intégrer une agence spécialisé dans la rédaction ou il peut aussi évoluer en freelance. (site Le codeur, https://www.codeur.com/blog/le-metierde-redacteur-web/5).

7 Le texte rédigé par le rédacteur web ne semble a priori pas ancré dans une sphère d'activité ${ }^{6}$ précise, ce dont témoignent les observations que nous avons effectuées sur le terrain (voir aussi infra, 2.) et les définitions recueillies sur le web ou dans l'un des ouvrages spécialisés que nous convoquons ici, considéré comme une référence dans le 
domaine de la rédaction web $: \underline{\text { Bien rédiger pour le web : stratégie de contenu pour améliorer }}$ son référencement naturel ${ }^{7}$.

Ce qui va caractériser la rédaction web, c'est en fait la mise en œuvre au sein même d'une sphère d'activité (le tourisme, l'édition, la culture musicale) d'un certain nombre de compétences liées à la textualité numérique en même temps que des compétences rédactionnelles et cognitives plus classiques, que l'on observe dans la rédaction de textes non numériques (et en particulier pour tout texte de communication et de vulgarisation). Les définitions relevées indiquent l'adaptation d'un texte à des objectifs de communication, des capacités de veille informationnelle et de synthèse, des compétences stylistiques - comme écrire un "contenu clair, concis, dynamique » (Le codeur, ibid.), " avoir une bonne plume ", savoir composer un texte cohérent - enfin des compétences linguistiques comme la maitrise de la langue et la maitrise de différents répertoires linguistiques: " son français est irréprochable et il sait adapter son niveau de langage au public ciblé» (Journal du net). Si ce n'est pas toujours explicitement formulé, on comprend aussi à la lecture de ces présentations qu'il faut être doté d'un certain capital culturel (l'Onisep indique par exemple parmi les qualités requises « une solide culture générale »).
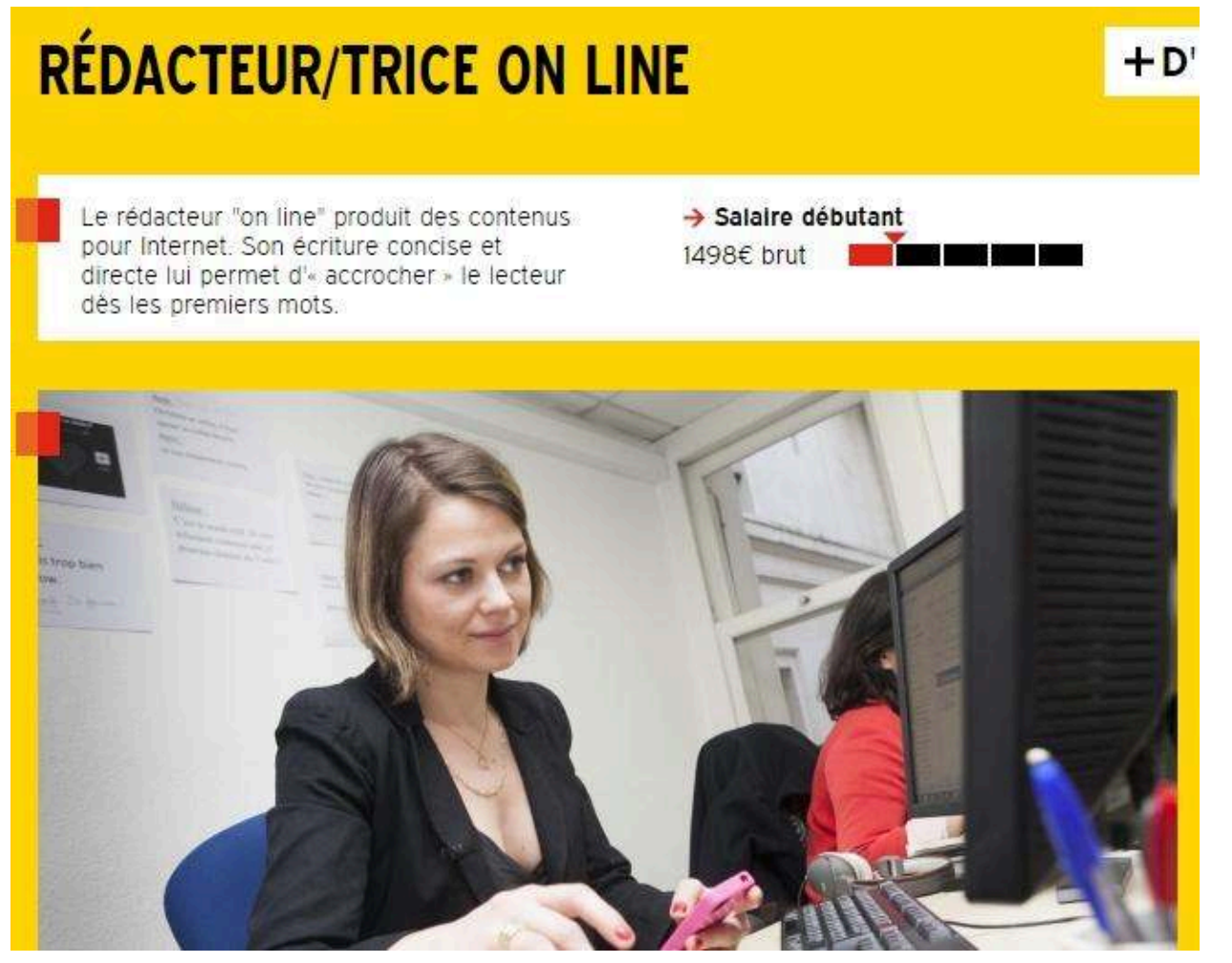


\section{En quoi consiste ce métier ?}

Articles sur le dernier concert de Lady Gaga, une naissance au zoo de Beauval, un match de la Coupe Davis, des produits high tech : les themes les plus varies sont traites sur la Toile

Le redacteur on line recueille des informations, les verifie et les rend accessibles au public. Pour traiter une information, il choisit un angle et s'efforce de capter l'attention du lecteur en utilisant un style direct et vivant. Qualites requises : une solide culture generale, une bonne plume, de l'aisance dans les relations humaines, de la debrouillardise, une curiosite toujours en eveil.

Les debouches sont tres diversifies : presse en ligne (generaliste ou specialisee), webzines (autour de centres d'interêt comme la musique, le tatouage...), journal d'entreprise, de collectivite territoriale, etc.

Extrait de la présentation par l'Onisep du métier de rédacteur/trice online

Dans les faits, les rédacteurs web que nous avons pu rencontrer sont souvent spécialisés dans un domaine (le tourisme, l'infodivertissement, la culture, la formation par exemple), suivant leurs centres d'intérêts, et peuvent être dotés d'un capital culturel les amenant à privilégier telle sphère plutôt que telle autre (voir aussi infra, 2.).

\subsection{Des textes ancrés dans des environnements numériques spécifiques}

Dans la représentation qu'offrent les professionnels du web du texte numérique, le terme même texte ne suffit pas. Lui est préféré le terme contenu, traduction de l'anglais content. C'est ce mot qu'utilisent un certain nombre de sites spécialisés dans le numérique ou l'orientation professionnelle (Journal du net, Le codeur, Onisep, par exemple) et que propose Isabelle Canivet (op. cit.) :

Penser en termes de contenu, pas seulement de texte.

La rédaction web doit se penser en termes de création de contenu et non de texte.

En effet, un texte publié sur l'Internet fait partie d'une page HTML qui intègre des éléments rédactionnels, des images, du son, de l'interactivité. Ce contenu se construit par le lien hypertexte et s'insère dans une structure globale. Il est impensable qu'un rédacteur web travaille coupé du site (dans son traitement de texte par exemple), sans en connaître l'architecture ni l'interface.

11 Si le livre traite effectivement de rédaction web, nous emploierons le plus souvent le terme "contenu" pour désigner tous les éléments d'information. (p. 8)

Ainsi conçu et nommé, le texte est un fragment ${ }^{8}$ ancré dans le dispositif et l'environnement technique et resitué dans un espace et une interface plurisémiotiques intégrant des éléments hybrides et hétérogènes mais formant un tout structuré.

Dans la pratique (voir infra, 2.), une part de cette hétérogénéité est directement gérée par le rédacteur web qui est tout à la fois scripteur, agenceur et éditeur de " contenus » (images, textes, titraille, liens...). Par contre, le rédacteur peut être cantonné à la production de certains types de textes (qu'il écrit «à la chaîne $»^{9}$ ), et non à une page dans son intégralité, ainsi qu'au suivi de ces textes sur les réseaux sociaux dont il a en quelque sorte la «responsabilité éditoriale». Ce qu'observent Étienne Candel et al. (2012, p. 19) pour les "petites formes", à savoir "un mouvement important de taylorisation dans les écrits d'écran $»^{10}$, peut s'appliquer ici dans une certaine mesure. 

d'une recherche sur les CMS ou "content management systems" (systèmes de management des contenus), Valérie Jeanne Perrier (2006) analyse le terme contenu comme un

terme suffisamment lisse pour qu'il désigne aussi bien des informations d'actualité destinées à éclairer l'opinion publique, des écrits sociaux destinés à rencontrer d'autres individus aux centres d'intérêts communs, des données à destination des militants ou des documents pour des populations plus ou moins homogènes dans différents types d'organisations » (p. 121).

Comme elle le souligne très justement, cette dénomination vague

permet également de s'éloigner de la parenté, lourde, avec le monde des médias. Il autorise une mise à distance des prérequis éthiques et politiques du travail de l'information, au sens journalistique de l'expression, tout en en conservant, en guise de caution de validité la technicité organisationnelle (p. 121-122).

\subsection{Des textes facilement « investigables », adaptés aux moteurs de recherche}

Par rédaction web, je fais référence à l'adaptation de l'écriture au média internet tout en tenant compte de l'influence et des répercussions de celle-ci sur le référencement. Son leitmotiv : bon pour les lecteurs, bon pour les moteurs ! (nous soulignons) (Canivet 2014, p. 2).

Dans les différentes définitions recueillies, le référencement ${ }^{11}$ se présente comme un enjeu et une contrainte forte de l'écrit et des textes produits ${ }^{12}$, faisant parfois presque oublier les autres spécificités des matérialités et textualités numériques (Paveau 2015, 2017, De Angelis 2018). Le texte rédigé pour le web est dès lors envisagé comme un contenu « optimisé » :

Qu'entend-on par optimiser? Il s'agit d'adapter le contenu à la manière dont les moteurs traitent l'information afin qu'ils identifient plus efficacement la pertinence du contenu en rapport à un sujet.

Pour ce faire, le rédacteur web adoptera certains réflexes d'écriture : chercher des mots-clés et les intégrer à des endroits stratégiques dans la page HTML, écrire des titres informatifs, renseigner les balises et les attributs, opter pour des noms de fichiers explicites, etc. Le rédacteur web est un acteur du référencement, à la fois par le fond et par la forme de son contenu. (ibid., 11)

17 Le texte rédigé par le rédacteur web est donc aussi conçu dans les discours professionnels comme un texte qui se doit d'être facilement «investigable " par les internautes, ou encore, il est surtout envisagé du point de vue de son investigabilité. Nous reprenons ce terme à Marie-Anne Paveau (2017, p. 29) pour désigner le fait qu'un matériau textuel numérique (ou un énoncé) soit « trouvable » et « collectable » (ibid.).

L'activité de référencement, qui est donc posée comme indissociable de la rédaction d'un texte pour le web, articule des compétences rédactionnelles et cognitives spécifiques de différents niveaux (synthétiser, identifier l'information importante, produire un type de discours informatif) et techniques (connaitre le code HTML, savoir modifier des balises). Dans les guides rédactionnels, un des aspects de cette activité, la recherche de mots-clés, figure parmi les premiers éléments d'élaboration du texte numérique, précédant la construction du plan, le choix de l'angle... Voici par exemple les étapes rédactionnelles préconisées par I. Canivet (op. cit.) :

Les clés de la rédaction:

Suivez les étapes ci-dessous avant même de commencer à rédiger : 
- définition de la cible et des objectifs

- identification des mots-clés [nous soulignons]

- choix de l'angle d'attaque

- rédaction du message essentiel

- choix du genre

- définition du plan de l'article. (p. 204)

\title{
Le rédacteur web et le SEO
}

Le rédacteur web doit idéalement avoir une autre compétence qui n'est pas exigée chez le journaliste : savoir optimiser ses contenus pour le référencement naturel (SEO). Pour faire simple, il doit être capable d'écrire des textes qui séduisent à la fois les internautes ET l'algorithme de Google.

-> Pour en savoir plus, lisez mon article "le SEO pour les nuls"

Extrait de l'article «Rédacteur web ou Journalisme, quelles différences ? » ${ }^{14}$, site Formation rédaction web

\section{Rédacteur web : description de l'emploi, formation, salaire...}

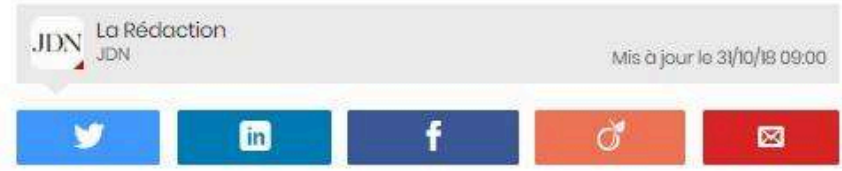

\begin{abstract}
Le rédacteur web rédige du contenu destiné à être publié sur internet ou dans des newsletters. Souvent spécialisé sur une thématique précise, il peut travailler pour le compte d'une agence, d'une entreprise ou en freelance. Découvrez comment devenir rédacteur Web, les formations à suivre, le salaire moyen...

Vos données ont un rôle à jouer

NATTENDEZ PLUS

Le rédacteur web doit ètre capable de rédiger du contenu sur un sujet précis tout en respectant certaines règles propres à lécriture web. Clair et concis, il donne un maximum dinformations en un minimum de mots et connait les bases du référencement. Les textes quil rédige sont optimisés pour les moteurs de recherche. Des connaissances en SEO, Search Engine Optimization, sont. indispensables, car un texte qui n'est pas visible par les moteurs de recherche ne génere pas de trafic et donc ne rapporte rienfe rédacteur web doit etre capable de faire des recherches efficaces sur le sujet traité, de synthétiser les informations recueilies et de répondre à une problématique en ajoutant une touche personnelle. Son français est irreprochable et il sait adapter son niveau de langage au public cible.
\end{abstract}

Extrait de l'article «Rédacteur web : description de l'emploi, formation, salaire... », Journal du net ${ }^{15}$

\subsection{Une écriture " efficace » et façonnée par les techniques d'écriture journalistiques}

21 Tels qu'ils sont représentés par les discours professionnels ou spécialisés dans le web, les textes issus de la rédaction web doivent à la fois avoir la propriété de pouvoir être 
vus, repérés facilement, de retenir l'attention du lecteur, en s'adaptant notamment à ses pratiques et ses besoins, et de déclencher une action (le plus souvent commerciale ${ }^{16}$ ). Tout comme pour les formes sémiotiques de la textualité numérique observées par Candel et al. 2012, c'est une « vision fonctionnaliste » et «pragmatique » (ibid., p. 12) de l'écrit qui prévaut. Dans les différentes présentations, la fonction conative de ces textes est prédominante et ils sont dotés d'une finalité fortement incitative :

le but ultime du contenu est d'amener le visiteur à réaliser ce que vous attendez de lui. Le call to action doit être converti en actions sonnantes et trébuchantes, on parle de conversion. Il peut aussi bien s'agir d'un achat, que de faire appel à vos services, ou d'une inscription à une newsletter, etc. (Canivet, op. cit., p. 8)

Passé aux cribles d'études marketing, le plus souvent quantitatives, le lecteur de ces textes est représenté comme un consommateur (d'écrits) à la fois vorace et frivole, recherchant la concision.

Sont alors convoquées et préconisées plusieurs techniques d'écriture journalistiques, qui concernent surtout l'organisation du contenu informationnel du texte (au niveau macro-textuel du plan de texte et au niveau micro-textuel de l'énoncé) et la titraille : le plan de la pyramide inversée ${ }^{17}$, la règle des $5 \mathrm{~W}^{18}$, et les multiples procédés pour créer des titres incitatifs. Il est ainsi remarquable de retrouver dans l'ouvrage de Canivet comme dans un guide plus ancien, écrit par Sébastien Bailly ${ }^{19}$, les mêmes techniques rédactionnelles que celles préconisées par des guides d'écriture journalistique (et en particulier, l'ouvrage de Jean-Luc Martin-Lagardette (2000 [1984] $)^{20}$.

Pour finir, le format court est roi («Plus c'est court, plus c'est bon », préconise S. Bailly (p. 26)), même si sur cet aspect les avis des professionnels semblent un peu plus nuancés voire contrastés, comment en témoigne l'extrait ci-dessous :

\section{Le texte est concis}

De manière générale, il cst courant de dire que le texte sur le Web doit être $50 \%$ plus court que sil ćtait publić sur papier. Ne prenons pas ces chiffres au pied de la lettre, mais tablons sur la richesse sémantique de la langue française pour gagner en concision. Quant aux recettes de cuisine toutes faites... bien que pitoyable cordon-bleu, je trouve qu'elles sentent souvent le brûlé.

Tableau 8-4 Le lectorat d'un texte en fonction de son volume

\begin{tabular}{|l|l|}
\hline 100 mots & Vous avez perdu $25 \%$ de vos lecteurs. \\
\hline 300 mots & Vous avez perdu $40 \%$ de vos lecteurs. \\
\hline 500 mots & Vous avez perdu $60 \%$ de vos lecteurs. \\
\hline 1000 mots & Vous avez perdu $80 \%$ de vos lecteurs. \\
\hline
\end{tabular}




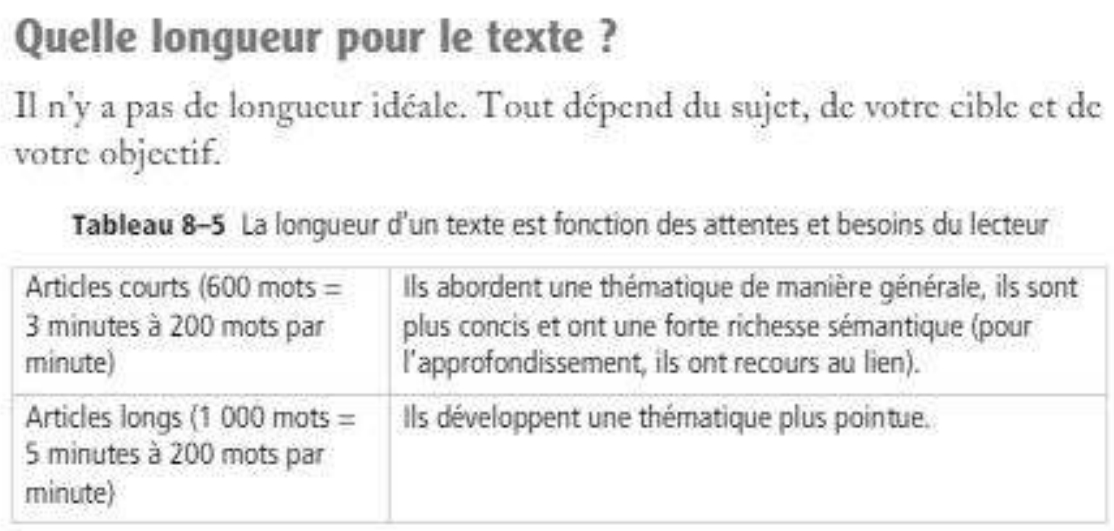

Extrait du manuel Bien écrire pour le web, I. Canivet, édition ebook, 2017, p. 261-262. qui ne se laissent pas observer de l'extérieur, mais requièrent une connaissance des dispositifs d'écriture et des cultures numériques, ainsi que des compétences dans les usages et les pratiques scripturales: les corpus numériques ne sont pas en effet des corpus parmi d'autres, mais plutôt des terrains, qui nécessitent la présence utilisatrice du chercheur. » (Paveau 2017, p. 132) Dans le cas de la rédaction web, la caractérisation des textes numériques nécessite aussi des enquêtes de terrain, à l'instar des enquêtes 
que l'on mène en anthropologie de l'écriture (cf. Borzeix et Fraenkel, 2001), et des investigations conduites par les chercheurs du Gripic.

C'est en faveur de cette double démarche que nous souhaitons plaider : considérer les textes de la rédaction web comme des terrains à investiguer et ce dans différentes sphères d'activité. Pour l'heure et de façon bien plus modeste, nous souhaitons confronter des pratiques d'écritures situées aux normes diffusées par les discours professionnels, et ce à partir de deux courtes illustrations de production d'écrits ${ }^{22}$. Il s'agit de mettre en lumière des caractéristiques du texte numérique (dans son versant discursif) et de la rédaction web qui ne transparaissent pas directement des discours professionnels que nous venons de parcourir.

Un premier exemple que nous nommerons désormais MUS porte sur un site spécialisé dans la culture et plus précisément dans un genre musical. Il propose des informations et des actualités sur le genre musical en question ainsi qu'une billetterie. Créé par un passionné venant d'un tout autre secteur, le site qui a le statut d'entreprise a bénéficié pour son lancement d'un financement privé. Des espaces publicitaires sont aussi proposés à des annonceurs (le plus souvent appartenant à la sphère musicale), ce qui constitue une source de revenu. Un deuxième exemple que nous appellerons TOU concerne un site spécialisé dans l'information touristique, rattaché à un groupe éditorial important. Le site TOU est le prolongement d'une collection de guides touristiques édités en format papier.

\subsection{Des textes spécialisés dans des domaines}

C'est une évidence, les textes produits pour les sites MUS et TOU appartiennent à des domaines distincts, la musique (un genre de musique spécifique) et le voyage. Dans le cas de MUS, la rédaction de textes implique une bonne connaissance du sujet et $a$ minima une acculturation. Un lexique spécialisé est employé dans tous les articles et il est important de connaître les artistes, les répertoires. Nous avons constaté que le site MUS employait des personnes qui avaient une formation musicale poussée et qui manifestaient un intérêt pour le genre musical en question.

Le cas du site TOU est peut-être un peu différent : on peut se dire que le voyage est un domaine qui n'implique pas des connaissances et un savoir spécifique et que le rédacteur peut développer progressivement une expertise. Cependant, la personne chargée de la rédaction web était passionnée par le voyage.

\subsection{Le poids de la ligne éditoriale}

En termes informationnels, le site MUS recherche l'exhaustivité et la mise à disposition d'une information individualisée pour le lecteur. Cela se traduit par la présence systématique de moteurs de recherche dans les différents espaces éditoriaux du site. Les textes sont donc accessibles via des rubriques, des liens et via ces "petites formes " (Candel et al., 2012) que sont les moteurs internes à un site.

35 Face à ses concurrents, le site essaye de se différencier en proposant une ligne éditoriale spécifique qui vise à offrir l'accessibilité et le partage de la culture musicale au plus grand nombre. Aussi, les textes produits, quelle que soit la rubrique, sont-ils caractérisés par une forte relationalité matérielle (Paveau 2017, p. 286), l'ensemble des noms propres mentionnés «ouvrant » sur des fiches descriptives. L'ensemble du site 
est en fait conçu comme une base de données à laquelle chaque texte est rattaché par plusieurs liens. De plus, les textes vont intégrer de nombreux matériaux visuels et/ou sonores qui éclairent un énoncé, la mention d'une référence. Enfin, cette ligne éditoriale entraîne des choix en termes de progression du texte. Par exemple, les écrits s'apparentant à des critiques de concerts, spectacles, vont systématiquement adopter une progression chrono-thématique, qui permet de « raconter » le spectacle au lecteur et de viser ainsi une communauté plus large.

Une partie de la ligne éditoriale du site TOU précède sa création, dictée par la ligne éditoriale des guides touristiques papier dont il est le prolongement. La précision et la qualité de l'information diffusée sont recherchées, ce qui implique, dans les textes rédigés pour le web, l'évitement de la reformulation (sauf pour des textes édités en format papiers dans les guides de l'entreprise) et la recherche d'un contenu original. Ce qui fait la spécificité du guide papier (le traitement de thèmes spécifiques, un discours subjectivisé, des énoncés embrayés, un répertoire linguistique...) va se retrouver dans les textes rédigés pour le web, de façon renforcée cependant (cf. infra).

Pour finir, la ligne éditoriale des deux sites est incarnée par des cadres d'écriture explicites, formulés à l'oral aux nouveaux arrivants, en amont, sous forme de conseils, ou en aval dans au moment où les textes produits sont relus.

\subsection{Des textes articulés et façonnés par des activités du site}

38 Du point de vue du rédacteur, l'identité des textes des sites est d'abord liée à leur ancrage dans un espace du site auquel sont associées des activités. Dans le cas de MUS il s'agit d'acheter un billet, de s'informer sur un spectacle/un groupe, de lire une critique, de s'informer sur un artiste/un groupe, d'écouter un morceau, de découvrir ou approfondir ses connaissances sur le genre musical traité. Certains textes du site sont circonscrits dans une rubrique spécifique: c'est le cas des textes de la billetterie. D'autres sont identifiés à la fois par leur appartenance à un même ensemble («les actualités ", par exemple) et par un nom spécifique. Par exemple, les écrits ayant trait à l'actualité musicale se subdivisent en plusieurs catégories pour le rédacteur du site, correspondant à des noms de genre bien établis dans la sphère journalistique ${ }^{23}$ : les «brèves », les « interviews », etc.

Dans le cas du site TOU, qui est structuré autour de plus d'une dizaine de rubriques ou d'espaces, il s'agit pour l'utilisateur de s'informer sur une destination, de rechercher des idées de voyage, de choisir une destination sur une carte interactive, d'acheter un guide, d'échanger avec des voyageurs, de réserver un voyage ou une prestation, etc. Les textes sont classés par rapport aux thèmes qu'ils abordent et l'on y retrouve les caractéristiques du discours touristique (Kerbrat-Orecchioni 2004, Seoane 2013), c'està-dire des textes à la fois descriptifs et évaluatifs. La variation entre ces textes va concerner surtout le degré d'interactivité et la place de l'iconographie plus ou moins importante.

\subsection{Un usage différencié des normes de la rédaction web}

Les deux sites MUS et TOU retiennent l'une des normes de la rédaction, la présence d'illustrations et de médias pour capter l'attention du lecteur. On note cependant quelques différences liées à la sphère d'activité. 
41 Les textes de MUS contiennent systématiquement des photos des artistes et des concerts ou spectacles. Celles-ci sont contraintes par la charte éditoriale et graphique du site (gabarit des photos, taille de la présentation) et paraissent indispensables aux rédacteurs pour des textes longs.

Le site TOU, quant à lui, accorde une très grande place aux images qui, nombreuses et de taille conséquente, doivent figurer comme un appel au voyage. Le premier accès au texte est souvent une image incrustée de texte, et certains " textes " ne sont en fait quasiment composés que d'images. Le domaine du tourisme autorise ici ce type «d'habillage » du texte ${ }^{24}$, de scénographie qui se situe dans une continuité par rapport aux textes touristiques prénumériques. Par rapport à MUS, d'autres stratégies discursives se mettent en place pour attirer le lecteur: le rédacteur mime une interaction, privilégie la modalité interrogative («Où part-on ce week-end? ») ou injonctive («Visitez...», « Découvrez...»), construit des énoncés courts, aphorisés (Maingueneau 2012), s'apparentant au slogan publicitaire, au proverbe, ou à la « petite phrase ». On peut alors relever des routines discursives en lien avec le domaine touristique.

43 L'incitation à l'action est formalisée dans les deux sites par la présence et la répétition de technomots en lien avec les domaines concernés : " réserver » pour le site MUS dans des textes de la billetterie, "réservations », noms de destination et URL vers des sites d'hôtels, d'offices du tourisme, etc. pour le site TOU.

Par rapport aux normes de la rédaction web, une différence importante se joue dans le degré de personnalisation du contenu à une catégorie de lecteur. Dans le cas de TOU, la rédaction des textes obéit à une stratégie de communication très informée des comportements des lecteurs. Un outil est utilisé, Google Analytics, qui livre des informations précises sur l'âge des internautes, le genre, le terminal utilisé pour lire les textes, les heures de consultation. Ces informations sont systématiquement analysées et exploitées pour la rédaction des textes et l'organisation générale du site, l'objectif étant d'être au plus proche du public qui fréquente le site et de ses pratiques de lecture sur le site.

On met au premier plan les types d'information ou les pages les plus consultées. Les énoncés produits tentent de s'adapter aux représentations que l'on peut se faire de ce public et à ses pratiques de lecture, ce qui a des implications lexicales, énonciatives, syntaxiques. Par exemple, les pages du site étant de plus en plus consultées sur des mobiles, les textes et les phrases des textes vont être raccourcis ${ }^{25}$. Ce sont donc des textes façonnés par les techniques du marketing qui sont proposés.

\subsection{Le référencement : un poids non négligeable mais variable suivant la sphère d'activité}

Le référencement intervient de manière sensiblement différente pour MUS et TOU. Certes dans les deux cas les rédacteurs éviteront la pratique de la reformulation, qui peut être pénalisée par le moteur de recherche Google. Pour améliorer la visibilité des textes et du site sur les moteurs de recherche, la relationalité matérielle du texte est aussi exploitée : par l'insertion de liens hypertexte dans les écrits (cf. supra), mais aussi par la publication systématique des textes sur les réseaux sociaux (Facebook et Twitter pour MUS, Facebook, Twitter, Instagram et Pinterest pour TOU). Les rédacteurs 
parsèment aussi le texte de noms susceptibles de correspondre à des requêtes sur les moteurs de recherche. Et c'est là que les pratiques se différencient. mérite une étude approfondie. C'est aussi une question qui semble importante pour les scripteurs ${ }^{27}$. Nous prendrons ici pour exemple des « critiques " publiés sur le site MUS. Il s'agit des textes les plus développés du site ( 800 mots environ auxquels s'ajoutent des vidéos de quelques minutes et des liens). Ces textes sont systématiquement signés. Malgré leur hétérogénéité sémiotique, ils se caractérisent par une forte unité thématique et sémantique, traitant de spectacles en particulier, et portent un nom de genre prénumérique pour les scripteurs comme pour les lecteurs. Ces textes s'apparentent au genre de la médiacritique (cf. Bertelli et Chauvin-Vileno éds, 2008) et sont identifiés comme tels par les scripteurs. Au sein de l'équipe du site MUS, le débat est d'ailleurs assez vif sur ce que doit être une critique. Les premières étapes de production de ces textes ${ }^{28}$ paraissent aussi très proches des pratiques du journalisme culturel : le scripteur se rend à un spectacle, prend des notes (sur un support papier), les restitue une fois rentré, rédige sur un terminal numérique une première version de la critique. Ce texte est ensuite envoyé aux rédacteurs du site MUS, qui procèdent à la relecture et l'enrichissent: imports des textes sur le back office du site, correction, ajouts d'illustrations (images, vidéos), de liens et de « tags » (ou mots-clés) ${ }^{29}$ permettant de les retrouver sur le site.

50 Ces critiques sont dans le même temps façonnées par la ligne éditoriale du site MUS : elles doivent à la fois informer, être accessibles, évaluer tout en n'étant pas trop dissuasives. Ces choix éditoriaux expliquent le plan adopté par la plupart des critiques publiées sur le site (cf. supra « Le poids de la ligne éditoriale ») : ces textes suivent une progression chrono-thématique, abordant les différents «moments» du spectacle, intègrent à plusieurs endroits des liens et des vidéos qui explicitent des aspects de la culture musicale traitée.

textes sont aussi relationnels, et ce à double titre : ils ouvrent sur d'autres textes mais ont aussi une "seconde vie» sur les réseaux sociaux, où ils sont systématiquement relayés via une page dédiée, donnant alors lieu à des réactions et 
commentaires qui "produisent un effet rétrospectif sur les unités premières [ici les critiques] et modifient donc leur significations » (Paveau 2017, p. 32). On peut parler ici à la suite de Paveau 2017 de « technogenre négocié »30 (p. 302).

\subsection{Le rédacteur web, un scripteur « tout terrain »?}

52 Une "économie » de la textualité numérique semble s'être mise en place dans de nombreuses webrédactions. Sur un laps de temps assez court, le rédacteur web peut être amené à produire des écrits et des textes de différents types, répondant à des visées pragmatiques très variées. C'est là peut-être l'une des spécificités de la rédaction web, qui absorbe dans une seule et même pratique d'écriture différents genres et formats d'écrits, appartenant dans un contexte prénumérique à des sphères différentes (cf. Jeanneret et Souchier, 2005). Ces textes peuvent impliquer un travail important de veille, de recherche d'information et de reformulation et une bonne connaissance culturelle dans le domaine concerné. Le rédacteur web est aussi mobilisé pour tout le versant technolangagier des textes produits (cf. supra). Enfin le travail iconographique peut occuper une place importante suivant les domaines et nécessite des compétences techniques et graphiques. Dans le cas du site MUS, il s'accompagne d'une activité de retouche (via des logiciels PAO) et entraîne des interactions avec les agents des artistes et les salles de spectacles. Dans le cas du site TOU, comme nous l'avons vu plus haut, le travail des illustrations est central dans la conception des textes. Un temps est réservé à l'exploration de banques d'images payantes (type Fotolia) et les photographies choisies sont ensuite systématiquement retravaillées.

53 Le rédacteur-web met aussi en ligne les textes et assure leur suivi sur les réseaux sociaux Facebook et Twitter (cf. supra, «Le référencement...»). Ces deux dernières activités s'apparentent plus à des missions éditoriales et obéissent à une organisation et un calendrier précis, informé par les pratiques des visiteurs des pages des sites sur les réseaux sociaux.

\section{Pour conclure}

54 Les discours professionnels sur la rédaction web indiquent un mouvement vers une très forte standardisation des textes numériques. Les textes paraissent ainsi construits sur le même modèle. Les deux exemples que nous avons abordés laissent en fait entrevoir des dynamiques entre des normes communes et des pratiques d'écritures liées à des domaines, des sphères d'activités et des enjeux économiques. Outre leur ancrage dans un lieu spécifique du site et les noms qui leur sont éventuellement attribués en amont, les textes numériques produits dans le cadre de la rédaction web sont ainsi façonnés par leur visée pragmatique et communicationnelle, la ligne éditoriale et la charte rédactionnelle du site qui les publie, les spécificités de la textualité numérique, les usages dans la sphère d'activité où ils se situent, enfin le genre auquel ils s'apparentent ou auquel ils empruntent. Surtout, les textes issus de la rédaction web paraissent pris dans une tension : ils doivent être repérés pour être lus et ont vocation, pour un certain nombre d'entre eux, d'inciter à l'action. Mais, dans le même temps, être lu (jusqu'au bout) implique de répondre aux attentes du lecteur, de s'inscrire pour cela dans des normes discursives (discours touristique, culturel) et génériques (critique musicale par exemple), et de figurer comme autant d'éléments d'un tout cohérent (le site internet). 
Pour le dire vite, ces textes sont à la fois conçus, écrits pour être "vus » (sur les moteurs de recherche) et écrits pour être «lus»... ce qui implique des usages différenciés de certains éléments spécifiques à la textualité numérique.

La pratique de la rédaction web nécessite quant à elle une culture de l'écrit et du numérique, une polyvalence et une adaptabilité, qui rappellent les compétences décrites par Jeanneret et Souchier (2005) concernant l'écrit d'écran :

La démocratisation annoncée par les idéologues se traduit finalement par un éclatement, une complexification des compétences d'écriture : en se propageant, mais sous des formes complexes, l'institution de l'image du texte appelle des compétences particulières, des lucidités particulières, que les programmes éducatifs ne prennent pas en charge et qui relèvent, ainsi, des habiletés et des opportunités. (2005, p. 13)

Pour finir, nous avons pu voir au cours de cette première tentative de caractérisation qu'une prise en compte fine des contextes et des modes de productions et, avec eux, de pratiques situées (dans des sphères économiques, professionnelles...) est indispensable à l'analyse de ces écrits dans une perspective discursive, c'est-à-dire à une analyse où les régularités formelles et points de rupture observés sont rapportés à un faisceau de déterminations de nature diverse : contraintes extérieures (Sitri, 2015), ou encore des lieux (Maingueneau 1998) et des cadres sociaux (Krieg-Planque et Bonnafous, 2013).

\section{BIBLIOGRAPHIE}

Adam Jean-Michel (2011). La linguistique textuelle: Introduction à l'analyse textuelle des discours, Paris : Armand Colin.

Adam Jean-Michel (éd.) (1997). « Genres de la presse écrite », Pratiques, 94.

Baudouin Valérie (2014). « Comment se constituent les genres à l'ère du numérique ? ", in Ablali Driss, Badir Sémir, Ducard Dominique (éd.) (2014) Documents, textes, œuvres. Perspectives sémiotiques, Rennes : PUR.

Borzeix Anni, Fraenkel Béatrice (dir.) (2001). Langage et travail. Communication, cognition, action, Paris : CNRS Editions.

Candel Étienne, Jeanne Perrier Valérie, Souchier Emmanuël (2012). « Petites formes, grands desseins. D'une grammaire des énoncés éditoriaux à la standardisation des écritures ", in J. Davallon Jean (dir.), L'économie des écritures sur le web, volume 1 : Traces d'usage dans un corpus de sites de tourisme, Paris : Hermès-Lavoisier, p. 165-201.

Canivet Isabelle (2014). Bien rédiger pour le web : stratégie de contenu pour améliorer son référencement naturel, Paris : Eyrolles, 3e édition.

Canivet Isabelle (2017). Bien rédiger pour le web : stratégie de contenu pour améliorer son référencement naturel, Paris : Eyrolles, 4e édition, Ebook.

De Angelis Rossana (2018). « Textes et textures numériques. Le passage de la matérialité graphique à la matérialité numérique ", Signata. Annales des sémiotiques, 9/2018, p. 459-484. 
Devriendt Émilie (éd.) (2017). Dire les conflits de normes. Discours et enjeux critiques, Langage et société, 159.

Jeanneret Yves, Souchier Emmanuël (2005). "L'énonciation éditoriale dans les écrits d'écran", Communication et langages, 145, p. 3-15.

Gournay Lucie, Née Émilie (2016). « S'affranchir des normes de l'écrit académique par la réécriture ", Pratiques [En ligne], 171-172, http://journals.openedition.org/pratiques/3218

Kerbrat-Orecchioni Catherine (2004). « Suivez le guide ! Les modalités de l'invitation au voyage dans les guides touristiques : l'exemple de l'Ile d'Aphrodite », dans Fabienne Baider, Bonnafous Simone, Krieg-Planque Alice (2013). «L'Analyse du discours », in Olivesi Stéphane (dir.), Sciences de l'information et de la communication. Objets, savoirs, discipline, Grenoble : Presses universitaires de Grenoble, p. 223-238.

Goutsos Dyonisis, Burger Marcel, Baider Fabienne éds (2006). La communication touristique. Approches discursives de l'identité et de l'altérité, Paris : L'Harmattan, p. 133-150.

Laborde-Milaa Isabelle, Plane Sylvie, Rinck Fanny, Sitri Frédérique (2014). Dossier « La formation aux écrits professionnels : des écrits en situation de travail aux dispositifs de formation », Le Discours et la langue, 5 (2).

Maingueneau Dominique (2012). Phrases sans texte, Paris : Armand Colin.

Maingueneau Dominique (2016 [1998]). Analyser les textes de communication, Paris : Dunod.

Martin-Lagardette Jean-Luc, (2000 [1984]). Le guide de l'écriture journalistique. Concevoir, rédiger, présenter l'information, Paris : Syros.

Paveau Marie-Anne (2015). « En naviguant en écrivant. Réflexions sur les textualités numériques ", dans Adam J.-M. (dir.), Faire texte. Unité(s) et (dis)continuité, Besançon : PUFC, p. 337-353.

Paveau Marie-Anne (2017). L'analyse du discours numérique. Dictionnaire des formes et des pratiques, Paris : Hermann.

Jeanne Perrier Valérie (2006). « Des outils d'écriture aux pouvoirs exorbitants ? ", Réseaux, 137, p. $97-131$.

Rinck Fanny, Sitri Frédérique (2012). « Pour une formation linguistique aux écrits professionnels, Littéracies universitaires : Nouvelles perspectives ", Pratiques, 153-154, p. 71-84.

Seoane Annabelle (2013). Les mécanismes énonciatifs dans les guides touristiques. Entre genre et positionnements discursifs, Paris : L'Harmattan.

Sitri Frédérique (2015). Parcours en analyse du discours : enjeux et méthodes. Autour d'écrits professionnels, Synthèse pour l'HDR en sciences du langage.

Zinna, Alessandro (2016). "L'interface : un espace de médiation entre support et écriture », Actes du congrès de l'Association Française de Sémiotique, Université du Luxembourg, 1-4 juillet 2015, http://afsemio.fr/wp-content/uploads/Sens-etm\%C3\%A9diation.-A.-Zinna.pdf

\section{NOTES}

1. Les réflexions et travaux présentés dans cet article étant encore programmatiques, ce travail relève plus d'une note de recherche en cours que d'un article présentant des résultats validés.

2. Par texte, nous entendons pour le moment un tout cohérent qui peut ou non s'insérer dans un ensemble plus vaste (un site, une rubrique, un blog, etc.). 
3. Comme nous l'avons observé à plusieurs reprises, les entreprises offrent d'ailleurs au rédacteur web débutant des formations accélérées insistant par exemple sur le référencement. 4. C'est le cas notamment d'un certain nombre d'ouvrages pratiques rédigés par des consultants spécialisés dans la rédaction web.

5. Consulté le 10.01.19.

6. Nous empruntons ici la catégorie de « sphère (sociale) d'activité » à Frédérique Sitri (2015), qui la reprend à $\mathrm{M}$. Bakhtine et la systématise. Dans une conception bakhtinienne des productions verbales, des "types relativement stables d'énoncés " c'est-à-dire des "genres de discours " (Bakhtine, 1984) sont envisagés comme étant « " produits " par des "sphères sociales d'activité" " et donc " indissociables de la sphère d'activité où ils sont produits » (Sitri 2015, p. 105). La sphère d'activité est envisagée par F. Sitri comme un lieu d'inscription des genres de discours et de ce fait comme un élément déterminant la forme des énoncés élaborés.

7. Isabelle Canivet semple être reconnue comme une figure importante de la rédaction web, ce que nous avons pu notamment constater en discutant avec plusieurs professionnels de la rédaction web et du référencement. Une quatrième réédition de son ouvrage est parue en 2017.

8. Nous reprenons ce terme à Candel, Jeanne Perrier, Souchier, 2012.

9. Un cas extrême est illustré par des webrédactions de sites d'information ou de divertissement où l'espace de travail des nombreux webrédacteurs fait irrésistiblement penser aux chaînes de montage ou de production de grandes usines, l'activité d'écriture étant alors souvent réduite à une activité de « réécriture » au sens de stricte reformulation.

10. "Chacun des acteurs impliqués dans ces objets, auxquels on peut conférer le statut d'œuvre collective, récupère une petite partie de la production du processus d'écriture, lequel est subdivisé en différentes étapes qui sont appropriées par des métiers en devenir » (p. 19).

11. Le référencement peut être défini strictement comme le positionnement d'une page ou d'un site sur les moteurs de recherche. Lorsqu'il est question de rédaction web, que ce soit dans les manuels ou dans la pratique, c'est en fait l'algorithme de Google et les outils proposés par l'entreprise qui sont pris en considération (voir aussi infra, 2.). De plus, le positionnement d'une page ou d'un site est en relation directe avec les requêtes des internautes.

12. La deuxième partie du titre de l'ouvrage proposé par I. Canivet thématise d'ailleurs cet enjeu : Bien rédiger pour le web: stratégie de contenu pour améliorer son référencement naturel (nous soulignons).

13. Articles « Augmentation », « Délinéarisation », « Écriture numérique ».

14. https://formation-redaction-web.com/redacteur-web-journaliste-differences/, consulté le 10.01.19.

15. https://www.journaldunet.fr/management/guide-du-management/1199395-redacteur-webdescription-de-l-emploi-formation-salaire/, consulté le 15.01.19, nous avons surligné certains passages.

16. On retrouve là semble-t-il un aspect que décrivent Jeanneret et Souchier (2005) au sujet de l'industrialisation de l'écriture : «Cette industrialisation vise à élaborer des matrices de textes de nature à créer du profit, non par la dissémination des produits culturels, mais par la dissémination des moyens de production culturelle » (p. 14).

17. Ce plan « en entonnoir » consiste à aller du plus important au moins important.

18. Les $5 \mathrm{~W}$ renvoient à cinq questions auxquels doivent répondre les premières lignes d'un texte : Who ?, What ?, When ?, Where ?, Why? (lire par exemple Jean-Luc Martin-Lagardette, 2000, p. 38-40).

19. Bien écrire pour le web. Textes, images, publicités, Paris, Eyrolles, 2003.

20. On retrouve là, au niveau de la trame textuelle elle-même, l'emprunt de logiques et de pratiques issues du monde de la presse (cf. Valérie Jeanne Perrier, 2006).

21. Terme repris à Souchier, 1996. 
22. Les lignes qui suivent s'appuient sur trois types de matériaux : les mémoires professionnels d'étudiants qui ont été webrédacteurs pendant plusieurs mois, un entretien semi-directif mené auprès d'une ancienne étudiante (le 3 mai 2018, 40'), et nos propres observations sur les écrits produits et sur les lieux de stage. Pour des raisons de confidentialité, nous ne faisons pas figurer ici les noms des entreprises et des personnes concernées.

23. L'emprunt aux noms de genres journalistiques pour l'indexation des textes produits peut être en lien avec des représentations, et plus précisément avec la «noblesse » associée à la sphère culturelle et musicale et aux pratiques d'écriture concernées. Cela reste cependant à montrer dans une enquête ultérieure.

24. On retrouve semble-t-il ici un des mécanismes décrit par De Angelis (2018), à savoir que « le choix des matières des supports varie aussi selon la relation socialement établie entre le support portant le texte et le genre au sein duquel le texte s'inscrit [aussi] dont la médiation est assurée par le format » (en ligne). Ce qui est possible pour un texte d'information touristique est peutêtre moins envisageable pour une critique musicale. Lire aussi Zinna, 2016.

25. À l'inverse, les textes du site MUS seront de taille variable, en adéquation avec les différents genres auxquels ils empruntent : article d'information, textes promotionnels, médiacritique...

26. On observe avec les techniques de référencement une forte tendance à la nominalisation dans les écrits, qu'il serait intéressant à étudier de manière systématique.

27. Une ancienne étudiante devenue rédactrice web nous faisait part un jour de son inquiétude devant l'impossibilité à identifier le genre de tel ou tel texte numérique. Plusieurs étudiants ont aussi ressenti le besoin d'interroger dans leur mémoire professionnel l'identité générique des textes numériques qu'ils étaient amenés à produire pendant leur stage.

28. Nous avons reconstitué ces étapes à partir d'un entretien mené auprès de l'une des rédactrices web et contributrices du site.

29. Cet étiquetage permet ainsi un regroupement des textes au sein du site.

30. «Le technogenre négocié est un genre de discours préexistant et stabilisé ou non dans les productions prénumériques, mais qui se dote en ligne de traits proprement technolangagiers et technodiscursifs. Il n'est pas entièrement dépendant des outils numériques et circule dans les univers en ligne et hors ligne. » (p. 302).

\section{RÉSUMÉS}

Cette note de recherche prend pour point de départ un certain nombre d'observations empiriques sur les pratiques d'écritures relatives à la « rédaction web» (ou "webrédaction»). Son ancrage est celui de l'analyse du discours, envisagée comme discipline interprétative et lecture critique des énoncés mais aussi comme démarche susceptible d'apporter sa contribution à une didactique de l'écrit. Nous y livrons quelques premiers éléments de réflexion pour la caractérisation discursive des textes issus de la rédaction web, prenant en compte pour cela à la fois la composante discursive de ces productions et les discours construisant la rédaction web comme pratique d'écriture. A partir de deux exemples, nous montrons que les normes dominantes de la rédaction web, liées notamment aux contraintes du support (produire des écrits courts, par exemple) et à des enjeux économiques (être vu, repéré), sont diversement prises en compte suivant la sphère d'activité où sont produits les textes. 
This research note takes as its starting point a number of empirical observations on writing practices related to "web content writing". It is based on discourse analysis, seen as an interpretative discipline and critical reading of statements, but also as an approach that can contribute to the didactics of writing. We provide some initial elements of reflection for the discursive characterization of texts resulting from web content writing, taking into account both the discursive component of these productions and the discourses building web content writing as a writing practice. From two examples, we show that the dominant norms of web writing, linked in particular to the constraints of the medium (producing short writings, for instance) and to economic issues (being seen, identified), are variously taken into account in the different fields of activity in which the texts are produced.

\section{INDEX}

Mots-clés : analyse du discours, rédaction web, textualité numérique, pratiques d'écriture numérique, normes

Keywords : discourse analysis, web content writing, digital textuality, digital writing practices, standards

\section{AUTEUR}

\section{ÉMILIE NÉE}

Université Paris Est Créteil, CÉDITEC 